\title{
X-ray Spectroscopy of the Southwest Cygnus Loop
}

\author{
D. A. Leahy \\ University of Calgary, Alberta T2N 1N4, Canada
}

\begin{abstract}
The Cygnus Loop is one of the nearest supernova remnants $(440 \mathrm{pc})$ and has been studied extensively in radio through X-ray wavelengths. An unusual bright "V"-shaped region on the south-western limb (near RA $20^{\mathrm{h}} 46^{\mathrm{m}}$ and Decl. $+30^{\circ}$ ) was revealed in ROSAT PSPC observations of the Cygnus Loop. The left side of the "V" has one of the highest hardness ratios (0.9-2 keV / 0.1-0.4 keV) in the Cygnus Loop whereas the right side has one of the lowest. Images and spatially resolved spectra were obtained using Chandra ACIS observations. Some results of analysis of the images and spectra are presented here.
\end{abstract}

\section{Introduction}

The Cygnus Loop is a well-studied, nearby (440 pc; Blair et al. 1999), largediameter supernova remnant (SNR) with a clear shell-type morphology in radio, infrared, optical, and X-ray bands. Recent radio observations are presented in Leahy, Roger \& Ballantyne (1997) $(1420 \mathrm{MHz}$ continuum and polarization observations), Leahy \& Roger (1998) $(408 / 1420 \mathrm{MHz}$ and $1420 / 2695 \mathrm{MHz}$ spectral index variations), Leahy (2002) $(21 \mathrm{~cm}$ neutral hydrogen observations of the full Cygnus Loop region) and Leahy (2003) (detection and analysis of an expanding shell of neutral hydrogen along the northeast limb of the Cygnus Loop). The optical nebula is clearly visible on the Palomar sky survey plates. An optical and X-ray study of an optical knot on the SE rim of the Cygnus Loop is given by Graham et al. (1995), which also includes references to previous optical studies of the Cygnus Loop.

The southwest "V" region of the Cygnus Loop was first noted as unusual in the observations of Aschenbach \& Leahy (1999), which showed the left side of the "V" to be one of the hardest (in the ROSAT band) regions in the Cygnus Loop and the right side to be one of the softest. Figure 1 (left) shows the ROSAT 0.2-2.0 keV image of the Cygnus Loop from the ROSAT All-Sky-Survey. The southwest "V" is the bright region inside the western limb about two-fifths of the way up from the bottom of the image. Here are presented Chandra Advanced CCD Imaging Spectrometer (ACIS) observations of the area covering the "V".

\section{Data, Analysis, and Results}

A 10 ks exposure of the southwest "V" region was obtained with Chandra ACIS. All data processing was carried out using the CIAO v2.3 package. The ACIS image shows that the southwest "V" consists almost entirely of diffuse emission, with a few weak point sources contributing negligible amounts to the total emission. 

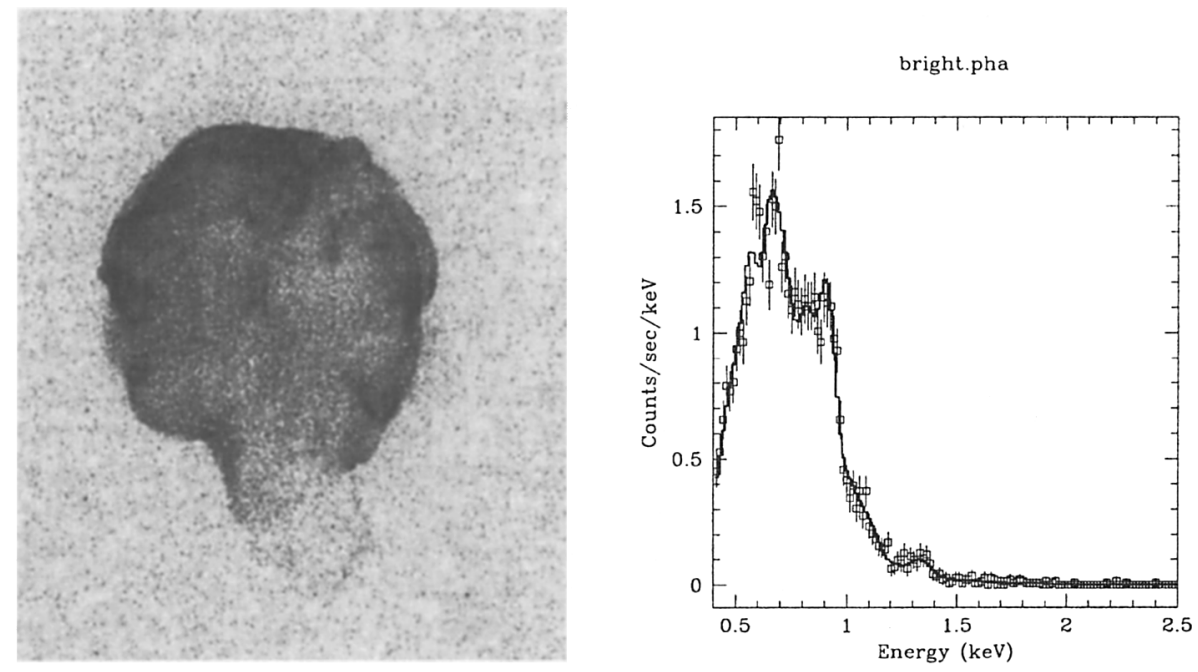

Figure 1. The ROSAT PSPC All-Sky-Survey image of the Cygnus Loop in the $0.2-2 \mathrm{keV}$ band (left); the Chandra ACIS spectrum of the bright region (right).

Spectral fitting was carried out on a number of sub-regions. One such region is the brightest subregion in the image from the center of the left side of the "V" (called "bright"). ACIS chip S3 was selected to determine the spectrum background emission. Several different source models were tried until a satisfactory one was obtained. A thermal plasma model (vmekal) with free $\mathrm{O}, \mathrm{Ne}$ and $\mathrm{Fe}$ abundances was satisfactory. Allowing non-equilibrium ionization did not improve the fits. Freeing other element abundances individually does not further significantly improve the fit $(<3 \sigma)$.

Comparing several regions, the temperature, column density, and $\mathrm{O}, \mathrm{Ne}$ and Fe abundances are all spatially variable.

Acknowledgments. This work supported by the Natural Sciences and Engineering Research Council of Canada.

\section{References}

Aschenbach, B., \& Leahy, D. 1999, A\&A, 341, 602

Blair, W., Sankrit, R., Raymond, J., \& Long, K. 1999, AJ, 118, 942

Graham, J., Levenson, N., Hester, J., Raymond, J., \& Petre, R. 1995, ApJ 444, 787

Leahy, D. A., Roger, R. S., \& Ballantyne, D. 1997, AJ 114, 2081

Leahy, D. A. \& Roger, R. S. 1998, ApJ, 505, 784

Leahy, D. A. 2002, AJ, 123, 2689

Leahy, D. A. 2003, ApJ, 586, 224 